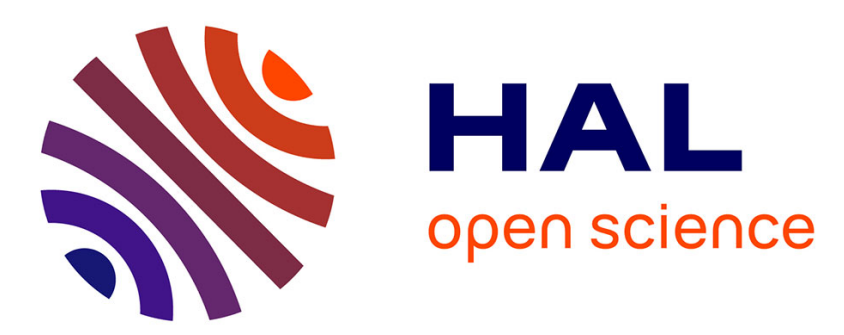

\title{
CONFIGURATION NON-COLINÉAIRE DES MOMENTS INDUITE PAR UN CHAMP MAGNÉTIQUE DANS LES FERRITES GRENATS DE TERRES RARES
}

G. Hug, P Morin

\section{To cite this version:}

G. Hug, P Morin. CONFIGURATION NON-COLINÉAIRE DES MOMENTS INDUITE PAR UN CHAMP MAGNÉTIQUE DANS LES FERRITES GRENATS DE TERRES RARES. Journal de Physique Colloques, 1971, 32 (C1), pp.C1-193-C1-194. 10.1051/jphyscol:1971158 . jpa-00214485

\author{
HAL Id: jpa-00214485 \\ https://hal.science/jpa-00214485
}

Submitted on 1 Jan 1971

HAL is a multi-disciplinary open access archive for the deposit and dissemination of scientific research documents, whether they are published or not. The documents may come from teaching and research institutions in France or abroad, or from public or private research centers.
L'archive ouverte pluridisciplinaire HAL, est destinée au dépôt et à la diffusion de documents scientifiques de niveau recherche, publiés ou non, émanant des établissements d'enseignement et de recherche français ou étrangers, des laboratoires publics ou privés. 


\title{
CONFIGURATION NON-COLINÉAIRE DES MOMENTS INDUITE PAR UN CHAMP MAGNÉTIQUE DANS LES FERRITES GRENATS DE TERRES RARES
}

\author{
G. HUG et P. MORIN \\ Laboratoire d'Electrostatique et de Physique du Métal, C. N. R. S., \\ Cédex 166, 38, Grenoble
}

Résumé. - Nous avous étudié le comportement magnétique d'un monocristal de ferrite grenat d'holmium HolG, suivant la direction [111], au voisinage de son point de compensation, dans des champs magnétiques qui atteignent $140 \mathrm{kOe}$. Sur les courbes d'aimantation isothermes, nous avons observé une discontinuité de la susceptibilité différentielle pour une valeur $H_{\mathrm{c} i}$ du champ appliqué. Ce comportement particulier de HoIG s'explique par un changement de configuration des moments : passage d'une structure colinéaire stable en champs faibles à une structure oblique stable en champs forts.

Abstract. - This paper describes the magnetic behavior of a single crystal of holmium iron garnet along the [111] axis, near its compensation point, in magnetic fields up to $140 \mathrm{kOe}$. On the magnetization curves we noticed a discontinuity in the susceptibility which occurs at a critical field $H_{\text {ci. }}$. This special behavior of HolG may be explained by the fact that the colinear ferrimagnetic structure becomes angled, which only occurs in a magnetic field.

Introduction. - Les ferrites grenats de terres rares de formule $\mathrm{R}_{3} \mathrm{Fe}_{5} \mathrm{O}_{12}$ possèdent la structure cubique $\mathrm{O}_{b}^{10}$. Les sites a et $\mathrm{d}$ sont occupés par les ions $\mathrm{Fe}^{3+}$, alors que les sites $\mathrm{c}$ le sont par les ions terres rares $\mathrm{R}^{3+}$. Il existe entre les ions $\mathrm{Fe}^{3+}$ des sites a et $\mathrm{d}$ une très forte interaction négative, qui tend à aligner les moments de ces ions antiparallèlement; les interactions $a-c, d-c$ et $c-c$ sont beaucoup plus faibles. Il en résulte que le champ effectif qui agit sur les ions $\mathrm{R}^{{ }^{3}+}$ est faible et que l'aimantation du sous-réseau terre rare dépend beaucoup du champ appliqué. Alors que les interactions d'échange tendent à rendre les aimantations des sous-réseaux antiparallèles, un champ magnétique extérieur tend à les aligner parallèlement. On conçoit alors qu'il peut exister une région du plan $(H, T)$ dans laquelle l'énergie est minimale quand les aimantations des sous-réseaux ne sont plus colinéaires avec le champ.

Nous décrivons le comportement d'un monocristal de ferrite grenat d'holmium dans des champs magnétiques qui atteignent $140 \mathrm{kOe}$, et qui sont donc comparables au champ d'échange auquel est soumis un ion $\mathrm{Ho}^{3+}$ de la part des ions $\mathrm{Fe}^{3+}$.

Résultats expérimentaux. - Nous avons effectué des mesures d'aimantation isothermes; la précision sur l'aimantation est de l'ordre de $5 \times 10^{-3}$.

Sur la figure 1 nous avons représenté deux courbes d'aimantation pour des températures inférieures à la température de compensation $T_{\mathrm{c}}(129 \mathrm{oK})$. Sur chaque courbe on distingue deux régions. En champs forts, l'aimantation est directement proportionnelle au champ appliqué; pour les différentes isotbermes, ce coefficient de proportionnalité est le même et vaut $23,5 \mathrm{kOe} / \mu_{\mathrm{B}} /$ formule $\mathrm{Ho}_{3} \mathrm{Fe}_{5} \mathrm{O}_{12}$; lorsque le champ décroît, la susceptibilité différentielle présente une discontinuité pour une valeur $H_{\mathrm{ci}}$ du champ appliqué ; lorsque le champ continue à décroître, on tend vers l'aimantation spontanée. Sur la figure 2 nous avons représenté la variation thermique de $H_{\mathrm{ci}}$ au voisinage de la température de compensation. On remarque sur la figure 1 que la discontinuité de la susceptibilité différentielle au champ critique est d'autant moins

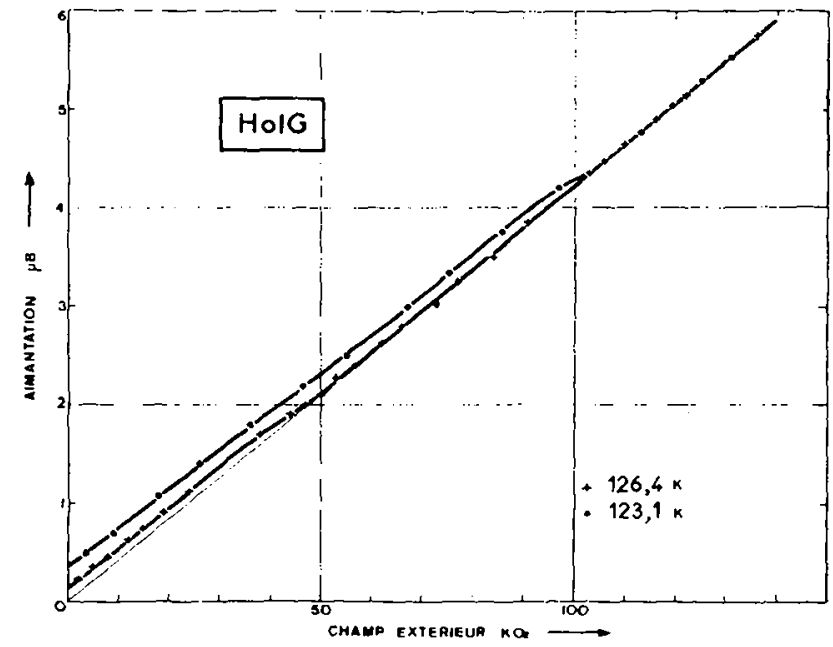

FrG. 1. - Courbes d'aimantation isothermes de HoIG pour des températures voisines du point de compensation.

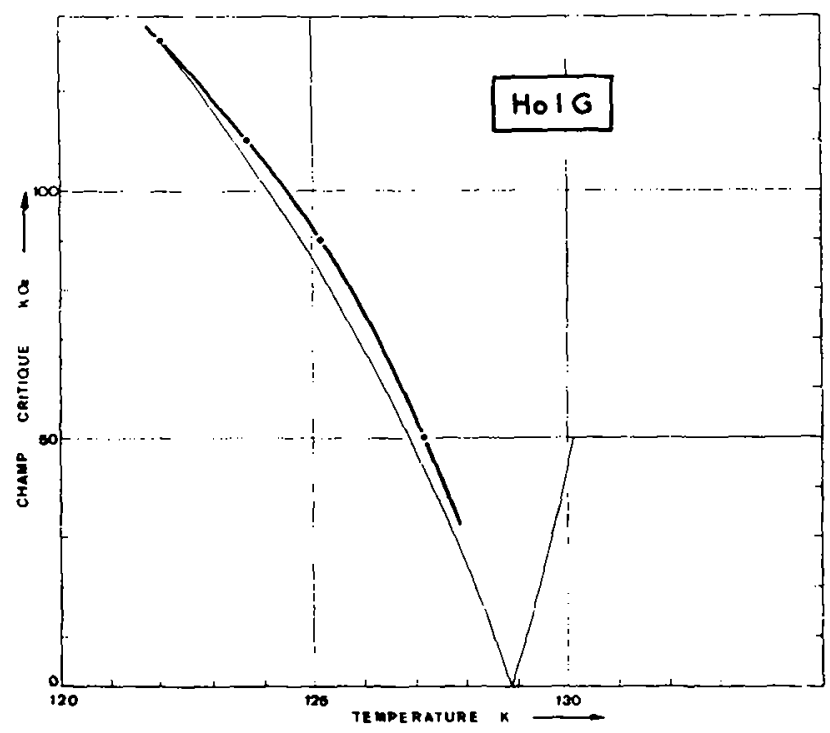

FIG. 2. - Variation thermique du champ critique $H_{c 1}$ (en trait fin : variation théorique). 
grande que la température est plus proche de $T_{\mathrm{c}}$; par suite, il n'est pas facile de déterminer $H_{\mathrm{ci}}$ dans cette zone de température. En outre, en raison des champs magnétiques continus disponibles, nous n'avons pu déterminer $H_{\mathrm{ci}}$ que dans un faible intervalle de température $\left(10^{\circ} \mathrm{K}\right)$ autour du point de compensation.

Interprétation des Résultats [1, 2]. - Nous supposerons que les deux sous-réseaux fer peuvent être traités globalement comme un seul sous-réseau d'aimantation $M_{\mathrm{F}}$. L'aimantation du sous-réseau terre rare sera notée $M_{\mathrm{R}}$. Dans l'approximation du champ moléculaire, les champs effectifs qui agissent sur les sous-réseaux fer et terre rare ont pour expression

$$
\begin{aligned}
& \mathbf{H}_{F}=\lambda_{F} \mathbf{M}_{F}+\lambda \mathbf{M}_{R} \\
& \mathbf{H}_{\mathbf{R}}=\lambda \mathbf{M}_{\mathbf{F}}+\lambda_{R} \mathbf{M}_{\mathbf{R}}
\end{aligned}
$$

où $\lambda, \lambda_{R}$ et $\lambda_{F}$ sont les coefficients de champ moléculaire.

La configuration stable des aimantations des deux sous-réseaux est obtenue en minimisant l'énergie du système, somme de l'énergie dans le champ extérieur et de l'énergie d'échange. Nous négligeons l'énergie d'anisotropie. Soient $\theta_{R}$ et $\theta_{F}$ les angles que font $\mathbf{M}_{R}$ et $\mathbf{M}_{F}$ avec $\mathbf{H}$. L'énergie totale s'écrit alors :

$$
\begin{aligned}
E=-\mathbf{H} \cdot\left(\mathbf{M}_{\mathrm{R}}+\mathbf{M}_{\mathrm{F}}\right)-\frac{1}{2}\left(\mathbf{H}_{\mathbf{F}} \cdot \mathbf{M}_{\mathrm{F}}+\mathbf{H}_{\mathrm{R}} \cdot \mathbf{M}_{\mathrm{R}}\right) \\
=-H\left(M_{\mathrm{R}} \cos \theta_{\mathrm{R}}+M_{\mathrm{F}} \cos \theta_{\mathrm{F}}\right)-\frac{\lambda_{\mathrm{R}} M_{\mathrm{R}}^{2}}{2}- \\
\quad-\frac{\lambda_{\mathrm{F}} M_{\mathrm{F}}^{2}}{2}-\lambda M_{\mathrm{R}} M_{\mathrm{F}} \cos \left(\mathbf{M}_{\mathrm{R}}, \mathbf{M}_{\mathrm{F}}\right) .
\end{aligned}
$$

Pour que l'énergie soit minimale, il est nécessaire que les 3 vecteurs $H, M_{R}$ et $\mathbf{M}_{F}$ soient situés dans un même plan. Des conditions d'équilibre $\partial E / \partial \theta_{\mathrm{F}}=\partial E / \partial \theta_{\mathrm{R}}=0$, on déduit que l'aimantation totale $\mathbf{M}=\mathbf{M}_{\mathbf{R}}+\mathbf{M}_{\mathbf{F}}$ est toujours colinéaire à $\mathbf{H}$; si on élimine $\theta_{\mathbf{F}}$ entre les 2 équations, l'angle $\theta_{\mathbf{R}}$ sera alors solution de l'équation :

$$
\sin ^{2} \theta_{\mathrm{R}}\left[\cos \theta_{\mathrm{R}}-\frac{\lambda^{2}\left(M_{F}^{2}-M_{\mathrm{R}}^{2}\right)-H^{2}}{2 \lambda M_{\mathrm{R}} H}\right]=0 .
$$

Cette équation possède trois solutions, deux d'entre elles sont colinéaires et correspondent aux structures ferri- et ferromagnétique des aimantations des deux sous-réseaux, la troisième est oblique ; l'énergie de la solution oblique est, quelle que soit la valeur de $H$, inférieure à l'énergie des deux autres; la structure oblique n'a cependant d'existence physique que pour des champs compris entre $H_{\mathrm{ci}}=|\lambda|\left|M_{\mathrm{R}}-M_{\mathrm{F}}\right|$ et $H_{\mathrm{cs}}=|\lambda|\left(M_{\mathrm{R}}+M_{\mathrm{F}}\right)$, car en dehors de cet intervalle $\left|\cos \theta_{\mathrm{R}}\right|$ serait supérieur à 1 .

Dans la zone oblique, l'aimantation totale $M$ est proportionnelle à $H$, la susceptibilité est égale à $1 /|\lambda|$; elle est donc indépendante de la température, ce que nous avons vérifié expérimentalement. On en déduit directement que le champ d'échange fer-terre rare au zéro absolu est de $118 \mathrm{kOe}$, valeur à comparer avec celles de Caspari [3] (125 kOe et $65 \mathrm{kOe})$ et celle de Pauthenet [4] (93 kOe). Expérimentalement, nous n'avons pu observer que le champ critique inférieur $H_{\text {ci }}$; nous avons évalué théoriquement $H_{\mathrm{ci}}(T)$ à l'aide du modèle proposé par Clark [2]. L'accord est relativement satisfaisant. Les premières déterminations expérimentales des champs critiques pour HoIG avaient été effectuées par effet Faraday [5].

Nous avons étudié aussi les ferrites grenats de terbium, de dysprosium et d'erbium. Le phénomène que nous venons de décrire peut êter mis en évidence d'autant plus facilement que la température de compensation est plus basse.

\section{Bibliographie}

[1] Schlömann (E.), Solid State Physics in Electronics and Telecommunications, $1960,3,322$.

[2] Clark (A. E.) et Callen (E.), J. Appl. Phys., 1968, 39,5972 .
[3] Caspari (M. E.), Kolcki (A.), KoICKi (S.) et Wood (G. T.), Phys. Letters, $1964,11,195$.

[4] Pauthenet (R.), Ann. Phys., 1958, 3, 424.

[5] Kharchenko (N. F.), Eremenko (V. V.) et Belyi (L. I.), Soviet. Phys., JETP, 1968, 26, 869. 